\title{
Avaliação dos possíveis fatores de risco para reabsorção radicular apical externa após tratamento ortodôntico
}

\section{Evaluation of possible risk factors for external apical root resorption after orthodontic treatment}

\author{
Luciana Quintanilha Pires Fernandes, ${ }^{1}$ Jonas Capelli Júnior, ${ }^{1}$ Karina de Paula Lopes Campos, ${ }^{2}$ Marcela Mendes Medeiros Michelon, ${ }^{2}$ Guaracilei Maciel \\ Vidigal Junior $^{3}$ \\ ${ }^{1}$ Departamento de Odontologia Preventiva e Comunitária, Faculdade de Odontologia, Universidade do Estado do Rio de Janeiro, Rio de Janeiro, RJ, Brasil \\ ${ }^{2}$ Departamento de Prótese, Faculdade de Odontologia, Universidade do Estado do Rio de Janeiro, Rio de Janeiro, RJ, Brasil \\ ${ }^{3}$ Departamento de Procedimentos Clínicos Integrados, Faculdade de Odontologia, Universidade do Estado do Rio de Janeiro, Rio de Janeiro, RJ, Brasil \\ - Os autores declaram que não há conflito de interesse.
}

\section{Resumo}

Objetivo: realizar uma revisão sobre possíveis fatores de risco para RRAE em pacientes submetidos ao tratamento ortodôntico. Material e Métodos: a pesquisa foi realizada no banco de dados MEDLINE, através do PubMed, usando a ferramenta de busca avançada, onde foram inseridas as seguintes palavras-chave, escolhidos segundo o Descritores em Ciências da Saúde (DeCS): ortodontia, reabsorção radicular, incisivo e polimorfismo genético, associado aos operadores boleanos "E" e "OU". A busca encontrou artigos de 1993 até 2016. Resultados: constatou-se que o tratamento ortodôntico é um fator de risco para RRAE, sendo os incisivos superiores os dentes mais acometidos. O sexo e a idade do paciente não parecem influenciar o grau de RRAE, assim como outros fatores clínicos e relacionados ao tratamento ortodôntico, com exceção do tempo de tratamento prolongado e aplicação de forças pesadas, que estão associados com maiores níveis de RRAE. Tratamento com extrações, uso de elásticos intermaxilares e diferentes polimorfismos genéticos apresentaram resultados conflitantes, não sendo possível definir a real relação com a RRAE. Diferenças metodológicas e populacionais entre os artigos avaliados podem explicar a divergência de resultados encontrada na literatura. Conclusão: apesar de diversos fatores intrínsecos, clínicos, genéticos e relacionados ao tratamento ortodôntico terem sido descritos na literatura, os mais frequentemente descritos como reais fatores de risco para RRAE são o tempo de tratamento ortodôntico prolongado e a aplicação de forças pesadas.

Palavras-chave: Ortodontia; Reabsorção da raiz; Incisivo; Polimorfismo genético.

\section{ABSTRACT}

Objective: this study aimed to review the research related to possible risk factors of external apical root resorption (EARR), associated with orthodontic treatment. Material and Methods: the MEDLINE database was explored, via PubMed, using the advanced search option. The keywords selected from the Medical Subject Headings (MeSH), used in combination with "AND" and "OR," were: orthodontics, root resorption, incisor, and genetic polymorphism. The research found articles from 1993 to 2016. Results: it was found that the orthodontic treatment can be considered as a risk factor of EARR, mostly affecting the upper incisors. Sex and chronological age, along with other clinical factors and those related to orthodontic treatment, do not seem to influence the degree of EARR, except the prolonged treatment time and heavy forces that are associated with increased amounts of EARR. Studies evaluating treatment with extractions, intermaxillary elastics, and different genetic polymorphisms showed contradictory results, restricting the determination of whether these factors can contribute to EARR. Methodological and population sample differences among the evaluated articles may explain the divergent results found in the literature. Conclusion: although various intrinsic, clinical, orthodontic, and genetic factors were described in the literature, the most common factors associated with increased values of EARR were prolonged treatment time and heavy forces during the orthodontic treatment.

Keywords: Orthodontics; Root resorption; Incisor; Polymorphism genetic.

\section{Introdução}

A reabsorção radicular é uma das consequências indesejáveis do tratamento ortodôntico, que acomete principalmente os incisivos superiores (figura 1). ${ }^{1,2}$ Apesar de $32 \%$ dos pacientes submetidos ao tratamento ortodôntico apresentarem reabsorção radicular maior que $3 \mathrm{~mm}$, apenas $2 \%$ a $5 \%$ destes pacientes apresentam reabsorção maior que $5 \mathrm{~mm} \cdot{ }^{2-4}$ Quando ocorre durante o movimento ortodôntico, a reabsorção é resultado de um processo inflamatório ${ }^{5}$ e, por acometer principalmente o contorno do ápice da raiz, é comumente chamada de reabsorção radicular apical externa (RRAE). ${ }^{6}$

Diversos fatores intrínsecos, clínicos e relacionados ao tratamento ortodôntico já foram associados com a etiologia da RRAE, como etnia, sexo, idade, tipo de má oclusão, forma da raiz, tempo de tratamento, força ortodôntica aplicada, tipo de aparelho, quantidade de movimentação dentária
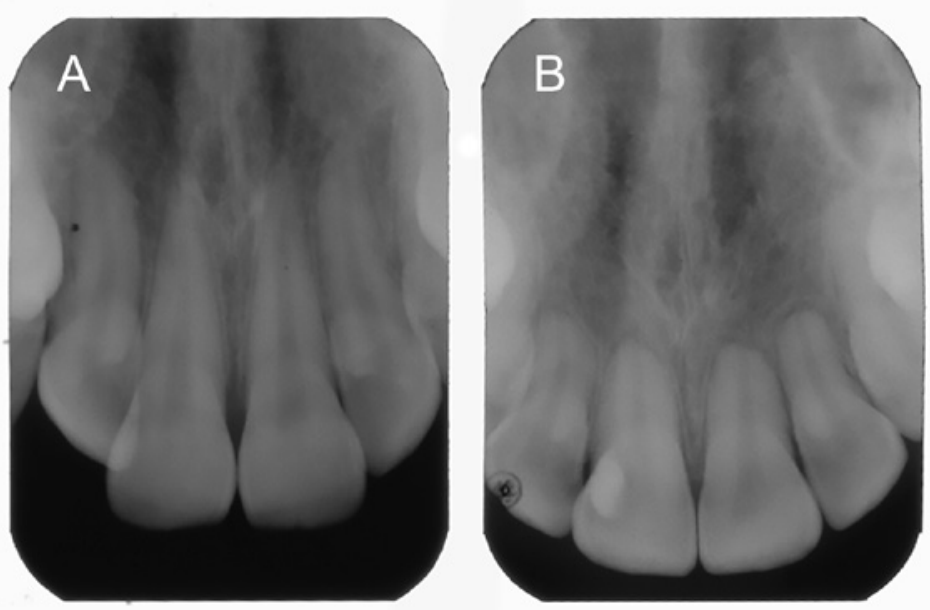

Figura 1. Radiografia periapical inicial (A) e final (B) dos incisivos superiores de uma paciente do sexo feminino, Classe I de Angle, tratada com aparelhos fixos e sem extrações dentárias. É possível observar uma significativa RRAE ao final do tratamento ortodôntico 
e tratamento ortodôntico com extrações. ${ }^{1,2,7-10}$ Entretanto, devido à divergência de resultados descritos na literatura, sugere-se que fatores genéticos também possam ter influência no desenvolvimento da RRAE após tratamento ortodôntico. ${ }^{11}$ Newman ${ }^{12}$ foi o primeiro a sugerir a susceptibilidade genética na etiologia da RRAE. Desde então, diversos estudos foram desenvolvidos avaliando se polimorfismos genéticos de citocinas e moléculas envolvidas no processo de RRAE também seriam um fator de risco para esta alteração patológica. ${ }^{6,13-27}$

Portanto, baseado na extensa literatura avaliando a associação entre RRAE e o tratamento ortodôntico, o objetivo deste estudo foi realizar uma revisão sobre os possíveis fatores de risco para RRAE em pacientes submetidos ao tratamento ortodôntico.

\section{Material e Métodos}

A pesquisa foi realizada no banco de dados MEDLINE, através do PubMed, utilizando-se o recurso de busca avançada. Foram utilizadas palavras-chave cadastradas nos Descritores em Ciências da Saúde (DeCS), com auxílio de operadores boleanos, da seguinte forma: ortodontia " $E$ " reabsorção radicular "E" incisivo "OU” polimorfismo genético.

A busca encontrou um total de 122 artigos, entre os anos de 1993 até 2016. Após avaliação dos títulos, foram selecionados 19 artigos que se aproximaram com o tema da revisão proposta por este estudo. Estes foram lidos na íntegra e, caso alguma referência utilizada nestes artigos fosse considerada de relevância para o presente trabalho, esta também foi incluída na revisão de literatura e na discussão descritas a seguir.

\section{Resultados \\ 1. Reabsorção Radicular Apical Externa (RRAE)}

Os odontoclastos são as principais células responsáveis pela reabsorção radicular e apresentam características citológicas e funcionais semelhantes aos osteoclastos, que também atuam no processo de reabsorção. Quando ocorre uma ruptura na região de pré-cemento, os osteoclastos entram em contato com os tecidos mineralizados e, assim, inicia-se o processo de reabsorção do tecido calcificado. ${ }^{28,29}$

Uma vez que o cemento radicular é mais resistente à reabsorção do que o tecido ósseo, a força aplicada com o tratamento ortodôntico gera uma reabsorção do osso, que permite a movimentação dentária sem danificar o cemento. Entretanto, uma vez que as células envolvidas no processo de reabsorção óssea também podem atuar no cemento e na dentina, pode haver reabsorção destes tecidos em alguns casos. ${ }^{28}$ Quando isto ocorre, pode-se observar a reabsorção de superfície ou inflamatória; raramente ocorre a reabsorção por substituição, que levaria à anquilose do dente. ${ }^{30}$

Fontana et al. ${ }^{6}$ relataram que os pacientes que utilizaram aparelho ortodôntico foram mais comprometidos (RRAE $+1,52 \mathrm{~mm}$ ) do que os pacientes não tratados (RRAE +0,05 $\mathrm{mm}$ ), ao estudarem a RRAE de incisivos superiores. De acordo com Killiany, ${ }^{3}$ RRAE maior que $5 \mathrm{~mm}$ poderia ocorrer em apenas $5 \%$ dos pacientes submetidos ao tratamento ortodôntico. Brin et al., ${ }^{31}$ em um estudo avaliando incisivos superiores após o tratamento ortodôntico, observaram RRAE moderada a severa em $12,4 \%$ da amostra, enquanto que em menos de $22 \%$ não foi possível detectar RRAE.

Apesar de Lee e Lee ${ }^{32}$ não terem encontrado diferença quanto ao grau de RRAE entre os diferentes dentes, alguns estudos já mostraram que os dentes superiores são mais suscetíveis à RRAE do que os inferiores. ${ }^{33}$ Os incisivos laterais superiores são descritos como os mais acometidos, ${ }^{24,34,35}$ assim como os incisivos centrais superiores. ${ }^{12,27,36,37}$

\section{Fatores Intrínsecos Relacionados com RRAE}

Diversos estudos não encontraram relação entre a idade de início do tratamento ortodôntico e o grau de RRAE ao final do tratamento. ${ }^{19,22-25}$ Entretanto, Artun et al. ${ }^{38}$ observaram maiores níveis de RRAE em pacientes com idade inicial reduzida, enquanto outros autores observaram que quanto mais velho o paciente iniciar o tratamento ortodôntico, maior será o grau de RRAE. ${ }^{6,32,35,39}$ Quanto ao sexo, a maioria dos estudos não observou diferença entre homens e mulheres. ${ }^{6,16,19,22,23,27,32,36,39}$ Em contrapartida, alguns estudos já mostraram maiores níveis de RRAE em homens ${ }^{24,25,40} \mathrm{e}$ outros em mulheres. ${ }^{41}$

\section{Fatores Clínicos Relacionados com RRAE}

A forma da raiz, sua largura, comprimento e estágio de formação já foram relacionados com grau de RRAE após uso de aparelho ortodôntico. Em geral, raízes mais longas e finas foram preferencialmente acometidas por RRAE. ${ }^{4,38}$ Fontana et al. ${ }^{6}$ observaram que incisivos superiores com comprimento de raiz maior que $30 \mathrm{~mm}$ apresentaram maiores níveis de RRAE do que raízes menores. Além disso, raízes com formato normal reabsorveram menos do que raízes com formato fora do padrão, ${ }^{31}$ como, por exemplo, raízes pontiagudas e dilaceradas. ${ }^{35,38}$ Entretanto, apesar destes estudos relacionando a morfologia radicular com a RRAE, uma revisão sistemática concluiu que esta variável não parece influenciar o grau de RRAE após tratamento ortodôntico. ${ }^{10}$

Pacientes com histórico de trauma nos incisivos superiores já foram avaliados quanto à magnitude de RRAE após tratamento ortodôntico e, apesar de alguns estudos defenderam a relação entre as duas variáveis, ${ }^{38,41,42}$ Weltman et al. ${ }^{10}$ realizaram uma revisão sistemática e concluíram que não há relação entre histórico de trauma anterior ao tratamento e RRAE após o aparelho ortodôntico. A presença de tratamento endodôntico em dentes que foram movimentados ortodonticamente exibiram menos RRAE do que dentes vitais e podem ser incluídos de forma segura no tratamento 
ortodôntico. ${ }^{32,43}$

Quanto às características dentárias da má oclusão, o overjet foi o mais associado com a magnitude de RRAE..$^{31,35,38}$ Maués et al. ${ }^{36}$ concluíram que um overjet maior do que 5 $\mathrm{mm}$ seria um fator de risco para RRAE após tratamento ortodôntico. Outras relações oclusais estudadas, como mordida aberta, ${ }^{24}$ overbite,${ }^{39,36}$ relação molar de Angle, ${ }^{19,22-25}$ desalinhamento ${ }^{12}$ e mordida cruzada ${ }^{12}$ não parecem ter relação com nível de RRAE.

Harris et al..$^{37}$ avaliaram medidas cefalométricas que poderiam estar associadas com RRAE e encontraram uma relação positiva para algumas destas medidas (ANB, FMA e SN-GoGn). Entretanto, estudos mais recentes não encontraram nenhuma associação entre RRAE e estas medidas cefalométricas. $^{24-26}$

\section{Fatores do Tratamento Ortodôntico Relacionados com RRAE}

Fontana et al. ${ }^{6}$ observaram que incisivos superiores de pacientes submetidos ao tratamento ortodôntico apresentaram maiores níveis de RRAE do que de pacientes que não haviam sido tratados. Weltman et al. ${ }^{10}$ realizaram uma revisão sistemática onde concluíram que o tratamento ortodôntico corretivo é um dos fatores etiológicos para RRAE. Além disso, muitos estudos já mostraram que quanto maior o tempo de tratamento com aparelho ortodôntico, maior será RRAE. ${ }^{23-26,31,32,36,39}$ Baumrind et al. ${ }^{40}$ concluíram que a cada ano a mais de tratamento, os incisivos superiores teriam mais $0,38 \mathrm{~mm}$ de RRAE. Uma revisão sistemática ${ }^{44}$ e uma meta-análise ${ }^{9}$ também concluíram que o tempo de tratamento prolongado estaria associado com maiores níveis de RRAE. Segundo Levander e Malmgren ${ }^{45}$ o grau de RRAE após os primeiros 6 a 9 meses de tratamento ortodôntico está significativamente relacionado com a quantidade de RRAE ao final do tratamento, sendo que os pacientes que apresentam uma RRAE suave neste período inicial, teriam um maior risco de desenvolver RRAE severa ao final do tratamento.

Não só o tempo de tratamento prolongado aumenta a RRAE, mas também a quantidade de deslocamento radicular gerada pelo aparelho ortodôntico ${ }^{9,40}$ e a aplicação de forças pesadas durante o tratamento. ${ }^{10,44,46}$ Em contrapartida, uma pausa de 2 a 3 meses durante o tratamento ortodôntico pode favorecer o processo de cicatrização do cemento de dentes que sofreram RRAE. ${ }^{10,44}$

Alguns estudos já observaram uma associação positiva entre tratamento realizado com extrações dentárias com finalidade ortodôntica e aumento de RRAE, ${ }^{6,8,24,25,34,36,38,39}$ enquanto outros não. ${ }^{18,19,20,22,23,26,27,32} \mathrm{O}$ uso de elásticos intermaxilares durante o tratamento ortodôntico apresenta resultados controversos, tanto para elásticos posteriores como anteriores. ${ }^{6,38,42}$ Para Motokawa et al., ${ }^{8}$ o uso de elásticos intermaxilares por mais de 6 meses seria um fator de risco para desenvolvimento de RRAE. Outras variáveis estudadas, como o tipo de aparelho, ${ }^{6,38,42}$ prescrição do bráquete ${ }^{10} \mathrm{e}$ tipo de fio ortodôntico ${ }^{10,38}$ não parecem influenciar o grau de RRAE ao final do tratamento.

\section{Fatores Genéticos Relacionados com RRAE}

Após Newman ${ }^{12}$ e Harris et al..$^{37}$ terem descrito que a RRAE apresenta um componente hereditário como fator etiológico, diversos estudos envolvendo questões genéticas foram desenvolvidos. O polimorfismo genético é uma variável bastante estudada, sendo definido como uma variação da forma do gene, que ocupa um determinado local no cromossomo, presente com frequência maior que $99 \%$ na população. ${ }^{47}$ Em geral, os estudos sobre polimorfismo apresentaram resultados divergentes, sendo necessárias mais pesquisas para melhor entendimento da real relação entre RRAE após o tratamento ortodôntico e as variáveis estudadas. ${ }^{48}$ Dentre os polimorfismos avaliados, estão os da IL-1 $\beta,{ }^{13,15,17,20-22,24-27,49}$ IL-1 $\alpha,{ }^{15,17,20,22}$ IL- $6,{ }^{16}$ IL-17, ${ }^{23}$ TNFa, ${ }^{14}$ TNSALP, ${ }^{14}$ TNFRSF11A, ${ }^{14,25}$ TNFRSF11B, ${ }^{23,25}$ OPG, ${ }^{25}$ OPN,,${ }^{19,23}$ CASP-1, ${ }^{26} \mathrm{P} 2 \mathrm{RX} 7,{ }^{23,25,26}$ receptor TaqI da vitamina $\mathrm{D}^{6}$, IRAK1 ${ }^{24}$ e IL-1RN. ${ }^{16,18,20,22,24}$

Dos estudos que avaliaram a relação de polimorfismos genéticos com RRAE, apenas dois foram realizados em amostra brasileira. Lages et al. ${ }^{21}$ observaram uma associação positiva entre o polimorfismo da IL-1 $\beta$ e RRAE, ao avaliarem radiografias periapicais de incisivos superiores de 61 brasileiros submetidos ao tratamento ortodôntico. Fontana et al., ${ }^{6}$ utilizando uma metodologia semelhante, observaram uma associação positiva entre o polimorfismo do receptor TaqI da vitamina D e RRAE, ao estudarem uma amostra de 377 brasileiros.

\section{Discussão}

Diversos fatores intrínsecos, clínicos, genéticos e relacionados ao tratamento ortodôntico já foram descritos na literatura, sendo a maioria deles com resultados conflitantes. Além da heterogeneidade da amostra, a diferença na metodologia utilizada em cada trabalho poderia justificar essa divergência de resultados.

A maioria dos estudos que não encontrou diferença quanto ao grau de RRAE entre homens e mulheres, assim como em relação à idade ao início do tratamento ortodôntico, utilizou radiografias panorâmicas para medir o comprimento total dos incisivos superiores, antes e após o tratamento ortodôntico..$^{19,22-25,27,32}$ Em contrapartida, Fontana et al. ${ }^{6}$ e Picanço et al..$^{39}$ encontraram valores maiores de RRAE em pacientes mais velhos ao início do tratamento, ao avaliarem incisivos superiores em radiografias periapicais. Além disto, outros estudos que utilizaram estas radiografias observaram resultados diferentes entre homens e mulheres. ${ }^{40,41}$ 
Sameshima e Asgarifar, ${ }^{50}$ ao avaliarem a forma radicular e a magnitude de RRAE em radiografias panorâmicas e periapicais, observaram que estas últimas são mais precisas, já que as radiografias panorâmicas podem supraestimar o grau de RRAE em $20 \%$.

Os resultados encontrados por estudos que avaliaram a RRAE utilizando tomografias computadorizadas ${ }^{16,34,46}$ poderiam ser considerados mais precisos, uma vez que a quantificação do grau de RRAE foi obtido por um método que permite uma avaliação tridimensional desta alteração patológica. Entretanto, por ser um exame com custo mais elevado e não fazer parte da documentação ortodôntica padrão, o tamanho da amostra desses estudos costuma ser mais reduzido. Enquanto Guo et al. ${ }^{16}$ conseguiram avaliar RRAE em uma amostra de 174 pacientes, utilizando tomografia computadorizada de feixe cônico, Sameshima e Sinclair ${ }^{35}$ avaliaram a RRAE após o tratamento ortodôntico de 868 pacientes, utilizando radiografias periapicais. Isto mostra que o uso de radiografias periapicais permite estudos com amostras maiores, o que seria uma vantagem em relação aos estudos que avaliam tomografias computadorizadas.

Apesar de Weltman et al. ${ }^{10}$ terem concluído que não há relação entre histórico de trauma anterior ao tratamento e RRAE após o aparelho ortodôntico, a partir de uma revisão sistemática, alguns autores já descreveram que estes dentes seriam mais propensos a desenvolverem RRAE após o tratamento ortodôntico. ${ }^{38,41,42}$ A divergência nos resultados poderia ser explicada pela falta de detalhes sobre o trauma que acometeu os dentes avaliados, como grau de comprometimento pós-trauma e tipo de tratamento que foi realizado nestes dentes, uma vez que estas variáveis poderiam influenciar no reparo tecidual no local.

Dentre os estudos que avaliaram a influência do tratamento ortodôntico envolvendo extrações dentárias no grau de RRAE, alguns avaliaram apenas incisivos ${ }^{6,22,23,26,27,36,38}$ e outros avaliaram também pré-molares e/ou caninos,, ,24,25,32,34 sem discriminar quais dentes sofreram RRAE. Além disso, alguns estudos informaram quais dentes foram extraídos durante o tratamento, $, 624,25,26,39$ enquanto os outros não. Tais diferenças metodológicas poderiam explicar as diferenças nos resultados, assim como a falta de padronização na mecânica e na força aplicada para fechamento de espaço da extração.

Outra variável relacionada com o tratamento que foi amplamente estudada foi o uso de elásticos intermaxilares, tanto posteriores quanto anteriores. Enquanto alguns autores afirmaram que o uso destes elásticos é um fator de risco para RRAE, ${ }^{8,38}$ outros não encontraram nenhuma relação entre ambas as variáveis. ${ }^{6,42}$ Os resultados não semelhantes poderiam ser explicados por se tratar de uma variável de difícil controle, uma vez que não é possível controlar a frequência de uso dos elásticos pelos pacientes, assim como a força exata que estes vão exercer sobre os dentes.

Em relação aos estudos envolvendo questões genéticas, um importante fator a ser observado é a origem étnica da população da amostra, uma vez que diferenças genéticas inerentes de cada população poderiam explicar as diferentes expressões de RRAE. ${ }^{21}$

\section{Conclusão}

O tratamento ortodôntico pode causar RRAE, sendo os incisivos superiores os dentes mais acometidos. Tempo de tratamento ortodôntico prolongado e aplicação de forças pesadas estão associados com maiores níveis de RRAE, enquanto outras características inerentes ao paciente e relacionadas com a má oclusão e o tratamento ortodôntico não parecem ter influência.

Diferentes polimorfismos genéticos parecem influenciar o grau de RRAE. Entretanto, os estudos genéticos devem ser avaliados com cautela, uma vez que diferenças inerentes de cada população podem influenciar nos resultados.

\section{Referências}

1. Brezniak N, Wasserstein A. Orthodontically induced inflammatory root resorption. Part II: the clinical aspects. Angle Orthod. 2002;72(2):180-4.

2. Hartsfield Junior JK, Everett ET, Al-Qawasmi RA. Genetic factors in external apical root resorption and orthodontic treatment. Crit Rev Oral Biol Med. 2004;15(2):115-22.

3. Killiany DM. Root resorption caused by orthodontic treatment: an evidence-based reviewof literature. Semin Orthod. 1999;5(2):128-33.

4. Taithongchai R, Sookkorn K, Killiany DM. Facial and dentoalveolar structure and the prediction of apical root shortening. Am J Orthod Dentofacial Orthop. 1996;110(3):296-302.

5. Liu Z, Xu J, Lingling E, Wang D. Ultrasound enhances the healing of orthodontically induced root resorption in rats. Angle Orthod. 2012;82(1):48-55.

6. Fontana MLSSN, Souza CM, Bernardino JF, Hoette F, Hoette ML, Thumet L, et al. Association analysis of clinical aspects and vitamin D receptor gene polymorphism with external apical root resorption in orthodontic patients. Am J Orthod Dentofacial Orthop. 2012;142(3):339-47.

7. Brezniak N, Wasserstein A. Root resorption after orthodontic treatment: part 2. Literature review. Am J Orthod Dentofacial Orthop. 1993;103(2):138-46.

8. Motokawa M, Sasamoto T, Kaku M, Kawata T, Matsuda Y, Terao A, et al. Association between root resorption incident to orthodontic treatment and treatment factors. Eur J Orthod. 2012;34:350-6.

9. Segal GR, Schiffman PH, Tuncay OC. Meta-analysis of the treatment-related factors of external apical root resorption. Orthod Craniofac Res. 2004;7:71-8.

10. Weltman B, Vig KWL, Fields HW, Shanker S, Kaizar EE. Root resorption associated with orthodontic tooth movement: a systematic review. Am J Orthod Dentofacial Orthop. 2010;137(4):462-76.

11. Hartsfield Junior JK. Pathways in external apical root resorption associated with orthodontia. Orthod Craniofac Res. 2009;12:236-42.

12. Newman WG. Possible etiologic factors in external root resorption. Am J Orthod Dentofacial Orthop. 1975;67(5):522-39.

13. Al-Qawasmi RA, Hartsfield Junior JK, Everett ET, Flury L, Liu L, Foroud TM, et al. Genetic predisposition to external apical root resorption. Am J Orthod Dentofacial Orthop. 2003;123(3):242-52.

14. Al-Qawasmi RA, Hartsfield Junior JK, Everett ET, Flury L, Liu L, Foroud TM, et al. Genetic predisposition to external apical root resorption in orthodontic patients: linkage of chromosome-18 marker. J Dent Res. 2003;82(5):356-60.

15. Gülden N, Eggermann T, Zerres K, Beer M, Meinelt A, Diedrich P. Interleukin-1 polymorphisms in relation to external apical root resorption (EARR). J Orofac Orthop. 2009;70(1):20-38.

16. Guo Y, He S, Gu T, Liu Y, Chend S. Genetic and clinical risk factors of root 
resorption associated with orthodontic treatment. Am J Orthod Dentofacial Orthop. 2016;150(2):283-9.

17. Iglesias-Linares A, Yañez-Vico RM, Ballesta S, Ortiz-Ariza E, Mendoza-Mendoza A, Perea E, et al. Interleukin 1 gene cluster SNPs (rs1800587, rs1143634) influences post-orthodontic root resorption in endodontic and their contralateral vital control teeth differently. Int Endod J. 2012;45:1018-26.

18. Iglesias-Linares A, Yañez-Vico RM, Ballesta S, Ortiz-Ariza E, Mendoza-Mendoza A, Perea E, et al. Interleukin 1 receptor antagonist (IL1RN) genetic variations condition post-orthodontic external root resorption in endodontically-treated teeth. Histol Histopathol. 2013;28:767-73.

19. Iglesias-Linares A, Yañez-Vico RM, Moreno-Fernández AM, Mendoza-Mendoza A, Orce-Romero A, Solano-Reina E, et al. Osteopontin gene SNPs (rs9138, rs11730582) mediate susceptibility to external root resorption in orthodontic patients. Oral Dis. 2014;20:307-12.

20. Iglesias-Linares A, Yañez-Vico RM, Ballesta S, Ortiz-Ariza E, Ortega-Rivera E, Mendoza-Mendoza A, et al. Postorthodontic external root resorption is associated with IL1 receptor antagonist gene variations. Oral Dis. 2012;18:198205.

21. Lages EMB, Drummond AF, Pretti H, Costa FO, Lages EJP, Gontijo AI, et al. Association of functional gene polymorphism IL-1b in patients with external apical root resorption. Am J Orthod Dentofacial Orthop. 2009;136(4):542-6.

22. Linhartova P, Cernochova P, Izakovicova Holla L. IL1 gene polymorphisms in relation to external apical root resorption concurrent with orthodontia. Oral Dis. 2013;19:262-70.

23. Linhartova PB, Cernochova P, Kastovsky J, Vrankova Z, Sirotkova M, Izakovicova Holla L. Genetic determinants and postorthodontic external apical root resorption in Czech children. Oral Dis. 2016:1-7.

24 Pereira S, Nogueira L, Canova F, Lopez M, Silva HC. IRAK1 variant is protective for orthodontic-induced external apical root resorption. Oral Dis. 2016;22:658-64.

25. Pereira S, Lavado N, Nogueira L, Lopez M, Abreu J, Silva H. Polymorphisms of genes encoding P2X7R, IL-1B, OPG and RANK in orthodontic-induced apical root resorption. Oral Dis. 2014;20:659-67.

26. Sharab LY, Morford LA, Dempsey J, Falcão-Alencar G, Mason A, Jacobson E, et al. Genetic and treatment-related risk factors associated with external apical root resorption (EARR) concurrent with orthodontia. Orthod Craniofac Res. 2015;18:71-82.

27. Tomoyasu Y, Yamaguchi T, Tajima A, Inoue I, Maki K. External apical root resorption and the interleukin-1B gene polymorphism in the Japanese population. Orthod Waves. 2009;68:152-7.

28. Brezniak N, Wasserstein A. Root resorption after orthodontic treatment: part 1. Literature Review. Am J Orthod Dentofacial Orthop. 1993;103(1):62-6.

29. Hammarstrom L, Lindskog S. General morphologic aspects of resorption of teeth and alveolar bone. Int Endod J. 1985;18:93-108.

30. Capelozza Filho L, Silva Filho OG. Reabsorção radicular na clínica ortodôntica: atitudes para uma conduta preventiva. Rev Den Press Orto Ortop Fac. 1998;3(1):104-26.

31. Brin I, Tulloch JFC, Koroluk L, Philips C. External apical root resorption in Class II malocclusion: A retrospective review of 1- versus 2-phase treatment. Am J Orthod Dentofacial Orthop. 2003;124(2):151-6.

32. Lee YJ, Lee TY. External root resorption during orthodontic treatment in root-filled teeth and contralateral teeth with vital pulp: A clinical study of con- tributing factors. Am J Orthod Dentofacial Orthop. 2016;149(1):84-9. 33. McFadden WC, Engstrom C, Engstrom H, Anholm JM. A study of the relationship between incisor intrusion and root shortening. Am J Orthod Dentofacial Orthop. 1989;96(5):390-6.

34. Freitas JC, Lyra OCP, Alencar AHG, Estrela C. Long-term evaluation of apical root resorption after orthodontic treatment using periapical radiography and cone beam computed tomography. Dental Press J Orthod. 2013;18(4):10412.

35. Sameshima GT, Sinclair PM. Predicting and preventing root resorption: Part I. Diagnostic factors. Am J Orthod Dentofacial Orthop. 2001;119(5):50510.

36. Maués CPR, Nascimento RR, Vilella OV. Severe root resorption resulting from orthodontic treatment: Prevalence and risk factors. Dental Press J Orthod. 2015;20(1):52-8.

37. Harris EF, Kineret SE, Tolley EA. A heritable component for external apical root resorption in patients treated orthodontically. Am J Orthod Dentofacial Orthop 1997;111(3):301-9.

38. Artun J, Hullenaar RV, Doppel D, Kuijpers-Jagtman AM. Identification of orthodontic patients at risk of severe apical root resorption. Am J Orthod Dentofacial Orthop. 2009;135(4):448-55.

39. Picanço GV, Freitas KMS, Cançado RH, Valarelli FP, Picanço PRB, Feijão CP. Predisposing factors to severe external root resorption associated to orthodontic treatment. Dental Press J Orthod. 2013;18(1):110-20.

40. Baumrind S, Korn EL, Boyd RL. Apical root resorption in orthodontically treated adults. Am J Orthod Dentofacial Orthop. 1996;110(3):311-20.

41. Linge BO, Linge L. Apical root resorption in upper anterior teeth. Eur J Orthod. 1983;5:173-83.

42. Linge L, Linge BO. Patient characteristics and treatment variables associated with apical root resorption during orthodontic treatment. Am J Orthod Dentofacial Orthop. 1991;99(1):35-43.

43. Ioannidou-Marathiotou I, Zafeiriadis AA, Papadopoulos MA. Root resorption of endodontically treated teeth following orthodontic treatment: a meta-analysis. Clin Oral Invest. 2013;17:1733-44.

44. Roscoe MG, Meira JBC, Cattaneo PM. Association of orthodontic force system and root resorption: A systematic review. Am J Orthod Dentofacial Orthop. 2015;147(5):610-26.

45. Levander E, Malmgren O. Evaluation of the risk of root resorption during orthodontic treatment: A study of upper incisors. Eur J Orthod. 1988;10:30-8. 46. Eross E, Turk T, Elekdag-Turk S, Cakmak F, Jones AS, Végh A, et al. Physical properties of root cementum: Part 25. Extent of root resorption after the application of light and heavy buccopalatal jiggling forces for 12 weeks: A microcomputed tomography study. Am J Orthod Dentofacial Orthop. 2015;147(6):738-46. 47. Schork NJ, Fallin D, Lanchbury JS. Single nucleotide polymorphisms and the future of genetic epidemiology. Clin Gent. 2000;58:250-64.

48. Aminoshariae A, Aminoshariae A, Valiathan M, Kulild JC. Association of genetic polymorphism and external apical root resorption: A systematic review. Angle Orthod. 2016. Versão online.

49. Wu FL, Wang LY, Huang YQ, Guo WB, Liu CD, Li SG. Interleukin- $1 \beta+3954$ polymorphisms and risk of external apical root resorption in orthodontic treatment: A meta-analysis. Genet Mol Res. 2013;12(4):4678-86.

50. Sameshima GT, Asgarifar KO. Assessment of root resorption and root shape: periapical vs panoramic films. Angle Orthod. 2001;71(3):185-9.

\section{Mini Currículo e Contribuição dos Autores}

1. Luciana Quintanilha Pires Fernandes - cirurgiã-dentista e especialista. Contribuição: participação científica e intelectual efetiva para o estudo; concepção e delineamento; aquisição dos dados; interpretação dos dados; preparação do manuscrito; redação do manuscrito; revisão crítica e aprovação final.

2. Jonas Capelli Júnior - cirurgião-dentista e PhD. Contribuição: participação científica e intelectual efetiva para o estudo; redação do manuscrito; revisão crítica e aprovação final.

3. Karina de Paula Lopes Campos - cirurgiã-dentista e especialista. Contribuição: participação científica e intelectual efetiva para o estudo; concepção e delineamento; interpretação dos dados; preparação do manuscrito; revisão crítica e aprovação final.

4. Marcela Mendes Medeiros Michelon - cirurgiã-dentista e especialista. Contribuição: participação científica e intelectual efetiva para o estudo; concepção e delineamento; interpretação dos dados; preparação do manuscrito; revisão crítica e aprovação final.

5. Guaracilei Maciel Vidigal Junior - cirurgião-dentista e PhD. Contribuição: participação científica e intelectual efetiva para o estudo; concepção e delineamento; interpretação dos dados; preparação do manuscrito; revisão crítica e aprovação final.

Recebido em: 14/12/2016 / Aprovado em: 15/03/2017

Autor Correspondente

Luciana Quintanilha Pires Fernandes

E-mail: lqpfernandes@hotmail.com 